\title{
Exploring Gender-Associated Socioeconomic Differences in Parkinson's Disease
}

\author{
Robert McInnis'1, William Cavanaugh1, Janice Weinberg'2, Marie-Hélène Saint-Hilaire ${ }^{1,3}$, \\ Samuel Ellias ${ }^{1,3}$, Samuel Frank ${ }^{1,3}$, Anna DePold Hohler ${ }^{1,3}$ \\ ${ }^{1}$ Boston University School of Medicine, Boston, MA, USA \\ ${ }^{2}$ Department of Biostatistics, Boston University School of Public Health, Boston University Medical Campus, \\ Boston, MA, USA \\ ${ }^{3}$ Boston Medical Center, Boston, MA, USA \\ Email: rmcinnis@bu.edu
}

Received 1 October 2015; accepted 14 November 2015; published 17 November 2015

Copyright (C) 2015 by authors and Scientific Research Publishing Inc.

This work is licensed under the Creative Commons Attribution International License (CC BY). http://creativecommons.org/licenses/by/4.0/

(c) (i) Open Access

\section{Abstract}

Background: Research on gender in Parkinson's disease (PD) frequently focuses on clinical differences between men and women. Socioeconomic factors such as race, ethnicity, insurance type, and level of educational attainment, have not been extensively examined in relation to gender differences in PD. The goal of this study was to identify differences in PD presentation in men and women, and identify socioeconomic factors that may confound such differences. Methods: A movement disorder patient database containing 445 patients with idiopathic PD was analyzed for gender differences in motor symptoms and disease complications using linear or logistic regression. Socioeconomic variables were then evaluated as possible confounders. Results: A greater proportion of women were non-white $(p<0.05)$. Univariate analysis of gender, and multivariate analysis controlling for age at diagnosis and socioeconomic factors were concordant in demonstrating increased frequency of motor fluctuations and dyskinesia in women $(p<0.05)$. Controlled, multivariate analysis, when compared to univariate analysis, uniquely demonstrated that women were less likely to experience dementia and autonomic dysfunction, relative to men $(p<0.05)$. Conclusion: Women with PD are susceptible to motor fluctuations and dyskinesia, but may be relatively protected against dementia and autonomic dysfunction. Women and men with PD in our population had different socioeconomic profiles, which may have confounded some gender-associated differences.

\section{Keywords}

Parkinson's Disease, Gender, Dementia, Social Determinants of Health

How to cite this paper: McInnis, R., Cavanaugh, W., Weinberg, J., Saint-Hilaire, M.-H., Ellias, S., Frank, S. and Hohler, A.D. (2015) Exploring Gender-Associated Socioeconomic Differences in Parkinson's Disease. Advances in Parkinson's Disease, 4, 84-89. http://dx.doi.org/10.4236/apd.2015.44010 


\section{Introduction}

Parkinson's disease (PD) is a neurodegenerative disorder whose incidence and clinical features are observed to differ between the sexes. In many populations, men are more frequently affected than women, with epidemiologic studies in Western nations reporting age-adjusted male to female ratios between 1.35 and 2 [1]-[4]. A meta-analysis performed by Taylor et al. demonstrated that Asian nations, including an Asian sub-population in California, observe more balanced ratios than Western populations [5]. So far, there have been no clear explanations for how regional and ethnic factors associate with sex in PD.

PD also presents with clinical differences between men and women. Studies in Dutch and Norwegian populations demonstrate that men are significantly younger at PD onset than women by about 2 years [6] [7]. Several studies found no difference in overall disease severity on the Unified Parkinson's Disease Rating Scale (UPDRS) in men and women at disease onset [7] [8] and in groups with mixed disease progression [1] [9]. Men have been found to have more rigidity [9], while women have more gait instability [9] [10]. Men experience sexual dysfunction [8] [11], fatigue [11], olfactory dysfunction [8] [11], and use caregivers more than women [12]. Dyskinesia has been widely reported to cluster in women [1] [13] [14], and women have been observed to experience dysphagia [15], restless legs [11], pain [11], and depressive symptoms more frequently than men [8] [11] [16].

This analysis examines a population of PD patients at an urban safety net hospital in Boston, MA to describe gender differences in PD within the context of socioeconomic factors such as racial and ethnic background, educational attainment, and insurance status. Often, clinical differences between men and women with PD are presumed to be a byproduct of sex, which refers to biological differences of men and women. Gender, in contrast, refers to the sociocultural factors by which men and women differ [17]. While the biology of sex differences in PD has been extensively examined, certain gender-based, socioeconomic differences likely contribute to the clinical picture as well, but are rarely the focus of research. Studying the patient population of a safety net hospital offers a valuable opportunity to characterize the socioeconomic factors related to PD.

\section{Methods}

\subsection{Patients and Location}

Patients were recruited from a movement disorders clinic in the neurology department at Boston University Medical Center (BUMC) in Boston, Massachusetts between 2007 and 2012. Patients entered into the database for this analysis were eligible if diagnosed by a movement disorder specialist at the clinic with idiopathic PD. New and existing patients without overlap were included in this analysis. The Institutional Review Board (IRB) at BUMC gave approval for this research.

\subsection{Data Collection}

Clinicians collected data using a standardized clinical characteristics data form. The data was then entered into a de-identified clinical database.

The data collection form included sections for demographics and clinical information. The demographics information included year of birth, sex, ethnicity, highest educational level completed, and insurance type. Clinical characteristics included the patient's diagnosis and date of diagnosis, as well as the date of symptom onset, as reported by the patient. Disease severity was characterized for each patient using the modified Hoehn and Yahr (HY) scale, both ON and OFF medication. This scale stages PD based on severity of motor symptoms, with Stage 1 representing unilateral motor symptoms only, and Stage 5 representing a wheelchair-bound or bedridden state [18]. The presence of several potential disease complications was also recorded in this section, which included compulsive behavior, dyskinesia, dementia, depression, hallucinations, motor fluctuations, orthostatic hypertension, freezing, psychosis, and other autonomic dysfunction, which refers to any autonomic dysfunction other than orthostatic hypotension.

\subsection{Data Analysis}

Four hundred and forty-five (445) patients were included in this analysis, whose demographic characteristics are outlined in Table 1. There were few non-white patients in our population, so patients were categorized as white or non-white. The majority of patients in our analysis were white (85.9\%), while African or African American 
Table 1. Demographic profile of patient population, with univariate comparison of men and women.

\begin{tabular}{|c|c|c|c|c|c|}
\hline Patient Demographics & Female & Male & $\begin{array}{l}\text { Whole } \\
\text { Population }\end{array}$ & Sample Size & $p$ Value* \\
\hline Number of patients (\%) & $184(41.3)$ & $261(58.7)$ & 445 & - & - \\
\hline Age in years (SD) & $68.6(11.8)$ & $68.2(10.4)$ & $68.3(11.0)$ & 441 & 0.712 \\
\hline Age at onset in years (SD) ${ }^{\dagger}$ & $59.2(12.6)$ & $57.4(10.7)$ & $58.2(11.6)$ & 355 & 0.147 \\
\hline Age at diagnosis in years $(\mathrm{SD})^{\dagger}$ & $61.1(10.7)$ & $59.1(10.9)$ & $59.9(10.9)$ & 369 & 0.08 \\
\hline Years since diagnosis (SD) & $7.58(6.7)$ & $8.39(7.2)$ & $8.01(7.0)$ & 371 & 0.274 \\
\hline Years between onset and diagnosis (SD) & $1.65(1.7)$ & $1.45(2.4)$ & $1.55(2.1)$ & 332 & 0.378 \\
\hline Educated beyond high school & $67.7 \%$ & $71.6 \%$ & $70.0 \%$ & 373 & 0.428 \\
\hline Public insurance $^{\dagger}$ & $69.5 \%$ & $60.6 \%$ & $64.3 \%$ & 403 & 0.067 \\
\hline Non-white ${ }^{\dagger}$ & $18.4 \%$ & $11.0 \%$ & $14.1 \%$ & 433 & 0.03 \\
\hline
\end{tabular}

SD = standard deviation, ${ }^{*} \alpha=0.05$ was threshold for statistical significance, while potential confounders of sex were identified using $\alpha=0.2$, ${ }^{\dagger}$ demarcates variables identified as potential confounders of sex.

patients (6.5\%), and Hispanic patients (3.7\%) comprised the next largest groups, respectively. Remaining racial and ethnic groups included Asian, Native American, Native Hawaiian or other Pacific Islander, and mixed race. These remaining groups altogether comprised $3.96 \%$ of the patient population. Level of educational attainment was also simplified for statistical analysis, dividing patients into those who had completed at least some college, and those with a High School diploma or less education. Each patient's insurance plan was classified as private or public (as of 2007 all patients were required by Massachusetts law to have insurance).

Among the socioeconomic variables, there were some missing data. Of the 445 patients included in this analysis, 90 had no age at onset, 76 had no age at diagnosis, 4 had no available age, and for 113, the number of years between onset and diagnosis could not be calculated. Seventy-two (72) patients were missing level of education information, 42 had no available insurance information, and 12 had no racial or ethnic information. Generally, data were missing because the study neurologists sometimes completed data collection forms after the patient encounter, and were unable to clarify the missing information once the patient had already left. After excluding those with missing data, 329 patients were included in the multivariate regression analyses.

Men and women were first compared using univariate analyses. T-tests and Chi-Square tests were used for continuous and categorical variables, respectively. For the analysis of socioeconomic variables, $\alpha$ was set to 0.2 , in order to screen out socioeconomic variables that were unlikely to be confounders, while remaining sensitive enough to identify variables that could confound the effect of sex. Since potential confounders were included in a multivariate regression model, it was optimal to limit the number of variables included, to achieve acceptable statistical power. For univariate analyses on the clinical variables of interest, as well as the multivariate regression analyses, $\alpha$ was set to 0.05 . Linear regression was used for continuous outcomes, with $\beta$ coefficients and standard error (SE) reported, while logistic regression was used for dichotomous outcomes, with odds ratios (OR) and confidence intervals (CI) reported. JMP statistical software (version 11) was used to run all statistical tests.

\section{Results}

\subsection{Analysis of Demographic Data and Confounders}

Women were more likely to be non-white compared to men $(18.4 \%, 11.0 \%$, respectively, $p=0.03)$. Men and women differed according to the confounder criterion $p<0.2$ on age at disease onset, in which women were on average 1.8 years older at onset $(p=0.147)$. A difference by this criterion was also observed for age at diagnosis, in which women were, on average, 2 years older at diagnosis than men $(p=0.08)$, and for insurance, in which women were more likely to be on public insurance than men $(69.5 \%, 60.6 \%$ respectively, $p=0.067)$. These tests established age at onset, age at diagnosis, insurance type, and race as possible confounding variables to include with sex in a regression model. Age at onset was excluded from the regression model, as it was too tightly linked to age at diagnosis to include both variables. Table 1 summarizes the demographic characteristics of our population, highlights significant results, and labels the variables identified as potential confounders. 


\subsection{Analysis of Clinical Outcomes}

On univariate analysis, women were found to have greater disease severity OFF medication on the HY scale, by 0.38 points $(p=0.0024)$. No difference, either ON or OFF medication, was observed for this measure on multivariate regression analysis. Univariate analysis also demonstrated that, among clinical complications, women more frequently experienced dyskinesia ( $55.4 \%$ vs. $31.4 \%, p<0.0001)$ and motor fluctuations $(60.3 \%$ vs. $44.1 \%, p$ $=0.0007)$, while men more frequently experienced freezing $(23.4 \%$ vs. $13.0 \%, p=0.0055)$. In comparison, multivariate regression analysis showed that women more frequently experienced dyskinesia $(p<0.0001$, OR $=2.92$, $\mathrm{CI}=1.78-4.85)$ and motor fluctuations than men $(p=0.004, \mathrm{OR}=2.07, \mathrm{CI}=1.26-3.42)$. Women were less likely to experience dementia $(p=0.005$, OR $=0.436, \mathrm{CI}=0.234-0.786)$ and other autonomic dysfunction $(p=$ 0.038 , OR $=0.513, \mathrm{CI}=0.263-0.964$ ) compared to men. Table 2 compares results from univariate and multivariate analysis of sex on clinical outcomes.

Table 2. Univariate and multivariate analyses on clinical outcomes.

\begin{tabular}{|c|c|c|c|c|c|c|}
\hline \multirow[b]{2}{*}{ Hoehn and Yahr Scores } & \multicolumn{3}{|c|}{ Univariate Analysis } & \multicolumn{3}{|c|}{ Multivariate Analysis } \\
\hline & Female & Male & $p$ Value $^{*}$ & $\beta$ Coefficient & Standard Error & $p$ Value $^{*+}$ \\
\hline ON medication (SD) & $0.755(1.48)$ & $0.705(1.33)$ & 0.713 & 0.006 & 0.077 & 0.934 \\
\hline OFF medication (SD) & $2.45(1.27)$ & $2.07(1.36)$ & 0.0024 & 0.103 & 0.071 & 0.145 \\
\hline Disease Complications & Female (\%) & Male (\%) & $p$ Value ${ }^{*}$ & Odds Ratio ${ }^{\ddagger}$ & $95 \%$ CI & $p$ Value $^{* \dagger}$ \\
\hline Compulsive behavior & 5.4 & 7.7 & 0.351 & 0.932 & $0.373-2.22$ & 0.875 \\
\hline Dementia & 17.9 & 23.4 & 0.164 & 0.436 & $0.234-0.786$ & 0.005 \\
\hline Depression & 23.9 & 19.5 & 0.269 & 1.33 & $0.789-2.26$ & 0.281 \\
\hline Dyskinesia & 55.4 & 31.4 & $<0.0001$ & 2.92 & $1.78-4.85$ & $<0.0001$ \\
\hline Freezing & 13 & 23.4 & 0.0055 & 0.552 & $0.292-1.01$ & 0.055 \\
\hline Hallucinations & 26.1 & 28.4 & 0.597 & 0.74 & $0.435-1.24$ & 0.257 \\
\hline Motor fluctuations & 60.3 & 44.1 & 0.0007 & 2.07 & $1.26-3.42$ & 0.004 \\
\hline Orthostatic hypotension & 13 & 12.3 & 0.807 & 1.15 & $0.568-2.32$ & 0.691 \\
\hline $\begin{array}{l}\text { Other autonomic } \\
\text { dysfunction }\end{array}$ & 12.5 & 17.2 & 0.167 & 0.513 & $0.263-0.964$ & 0.038 \\
\hline Psychosis & 3.8 & 2.7 & 0.507 & 1.62 & $0.468-585$ & 0.44 \\
\hline
\end{tabular}

$\mathrm{SD}=$ standard deviation, $\mathrm{CI}=$ confidence interval, ${ }^{*} \alpha=0.05,{ }^{\dagger}=p$ value for sex, adjusted for age at diagnosis, insurance type, and race, ${ }^{\ddagger}=$ compares women to men.

\section{Discussion}

We have demonstrated several sex-associated socioeconomic and clinical differences in this PD patient population. Men were more numerous in our cohort: the male to female ratio was 1.42, a distribution consistent with what is reported in Western societies [1]-[4]. Socioeconomic differences were also observed, in which men were more likely to be white, and tended to be privately insured, when compared to women. Possible explanations for this racial difference include a true increased incidence of PD in black women, a relative reduction in the rate that black men seek care for PD, or a difference in hospital patronage between men and women of different races, given the many medical centers located in this geographic region. Of note, a recent study in California demonstrated no racial differences in the comparative incidence of men and women with PD [4]. It is uncertain why more women tended to be on public insurance, as there were no age differences across gender and, by extension, no proportional differences in Medicare eligibility (most Americans become eligible for Medicare at age 65). A possible underlying factor may be differences in employment patterns between men and women with PD. A recent study on this topic demonstrated that PD patients who leave the workforce early are more likely to be female [19]. If this were true in our population, women would have a greater need for Medicare than men, as they would be less likely to be covered by an employer-based private insurance plan. 
Our data suggest that age at diagnosis, as well as socioeconomic factors such as insurance type and race, may confound gender-associated differences in PD. In particular, univariate analyses demonstrated that women experience greater disease severity OFF medication, and experience less freezing than men; however there was no difference with respect to these features when controlling for age at onset, insurance type, and race in a multivariate regression analysis. Therefore, we assume that one or several of these factors may confound the effect of gender on these outcomes. Further study is needed to delineate the individual contributions of these confounders. Univariate and multivariate regression analyses were concordant in showing that motor fluctuations and dyskinesia occurred more frequently in women, suggesting a true biologic difference, which has been demonstrated repeatedly in the literature [1] [13] [14]. Specifically, women have been found to achieve higher plasma concentration of levodopa after oral administration, leading to a greater incidence of motor side effects [13].

Controlling for confounders, multivariate analysis demonstrated that women are less likely to experience dementia and non-orthostatic type autonomic dysfunction. Studies on the relationship of gender with dementia in PD have been equivocal, showing increased frequency of dementia with PD in men in some populations [20] [21], while others find no differences [22] [23]. Non-orthostatic type autonomic dysfunction is an umbrella term that includes a wide variety of autonomic symptoms, including urinary and sexual dysfunction, gastrointestinal disturbances, and thermal dysregulation. Our data collection did not parse out these various symptoms; however, prior studies have demonstrated that sexual dysfunction is highly prevalent in men with PD [8] [11], which may underlie the difference in our population. However, other autonomic symptoms have been demonstrated to cluster in women with PD, such as constipation [11]. Further study is warranted in our patient population to better characterize these differences.

In this analysis, we observe that men and women with PD have different socioeconomic profiles, and that such differences may confound some of the clinical differences that associate with gender. In our cohort, age at onset, race, ethnicity, and insurance status were identified as potential confounders of gender in PD. Future research should examine these relationships, to identify any predictive value they may have for disease features. Our analysis also validates prior knowledge about sex differences in PD that, in particular, women are susceptible to levodopa-induced motor fluctuations and dyskinesia, and may be relatively protected from dementia and some autonomic disturbances. This analysis is particularly valuable for examining a large population at a safety net hospital, whose health outcomes are often associated with socioeconomic factors. Our analysis was limited by some missing socioeconomic data, lack of longitudinal follow-up, and that disease severity was measured solely by the HY scale, which describes only motor symptoms. More nuanced scales, such as the UPDRS, have been developed that evaluate a broader array of disease manifestations, such as mood, behavior, and functional capacity. A strong follow-up study could examine a diverse population of PD patients over time, to identify socioeconomic factors that may associate with poorer outcomes.

\section{Acknowledgements}

The authors thank Boston University School of Medicine and the Medical Student Summer Research Program for providing guidance and funding for this project.

\section{References}

[1] Accolla, E., Caputo, E., Cogiamanian, F., et al. (2007) Gender Differences in Patients with Parkinson's Disease Treated with Subthalamic Deep Brain Stimulation. Movement Disorders, 22, 1150-1156. http://dx.doi.org/10.1002/mds.21520

[2] Caslake, R., Taylor, K., Scott, N., et al. (2013). Age-, Gender-, and Socioeconomic Status-Specific Incidence of Parkinson's Disease and Parkinsonism in Northeast Scotland: The PINE Study. Parkinsonism \& Related Disorders, 19, 515-521. http://dx.doi.org/10.1016/j.parkreldis.2013.01.014

[3] De Lau, L.M.L., Giesbergen, P.C.L.M., de Rijk, M.C., et al. (2004) Incidence of Parkinsonism and Parkinson Disease in a General Population: the Rotterdam Study. Neurology, 63, 1240-1244. http://dx.doi.org/10.1212/01.WNL.0000140706.52798.BE

[4] Van Den Eeden, S.K., Tanner, C.M., Bernstein, A.L., et al. (2003) Incidence of Parkinson's Disease: Variation by Age, Gender, and Race/Ethnicity. American Journal of Epidemiology, 157, 1015-1022. http://dx.doi.org/10.1093/aje/kwg068

[5] Taylor, K.S.M., Cook, J.A. and Counsell, C.E. (2007) Heterogeneity in Male to Female Risk for Parkinson’s Disease. Journal of Neurology, Neurosurgery, and Psychiatry, 78, 905-906. http://dx.doi.org/10.1136/jnnp.2006.104695 
[6] Alves, G., Müller, B., Herlofson, K., et al. (2009) Incidence of Parkinson’s Disease in Norway: The Norwegian Park West Study. Journal of Neurology, Neurosurgery, and Psychiatry, 80, 851-857.

http://dx.doi.org/10.1136/jnnp.2008.168211

[7] Haaxma, C.A., Bloem, B.R., Borm, G.F., et al. (2007) Gender Differences in Parkinson's Disease. Journal of Neurology, Neurosurgery, and Psychiatry, 78, 819-824. http://dx.doi.org/10.1136/jnnp.2006.103788

[8] Picillo, M., Amboni, M., Erro, R., et al. (2013) Gender Differences in Non-Motor Symptoms in Early, Drug Naïve Parkinson's Disease. Journal of Neurology, 260, 2849-2855. http://dx.doi.org/10.1007/s00415-013-7085-X

[9] Baba, Y., Putzke, J.D., Whaley, N.R., et al. (2005) Gender and the Parkinson's Disease Phenotype. Journal of Neurology, 252, 1201-1205. http://dx.doi.org/10.1007/s00415-005-0835-7

[10] Solla, P., Cannas, A., Ibba, F.C., et al. (2012) Gender Differences in Motor and Non-Motor Symptoms among Sardinian Patients with Parkinson's Disease. Journal of the Neurological Sciences, 323, 33-39. http://dx.doi.org/10.1016/j.jns.2012.07.026

[11] Martinez-Martin, P., Pecurariu, C.F., Odin, P., van Hilten, J.J., Antonini, A., Rojo-Abuin, J.M., et al. (2012) Gender-Related Differences in the Burden of Non-Motor Symptoms in Parkinson's Disease. Journal of Neurology, 259, 1639-1647. http://dx.doi.org/10.1007/s00415-011-6392-3

[12] Lubomski, M., Rushworth, L.R., Lee, W., Bertram, K.L. and Williams, D.R. (2014) Sex Differences in Parkinson's Disease. Journal of Clinical Neuroscience, 21, 10-13. http://dx.doi.org/10.1016/j.jocn.2013.12.016

[13] Arabia, G., Zappia, M., Bosco, D., Crescibene, L., Bagalà, A., Bastone, L., et al. (2002) Body Weight, Levodopa Pharmacokinetics and Dyskinesia in Parkinson's Disease. Neurological Sciences, 23, S53-S54. http://dx.doi.org/10.1007/s100720200066

[14] Hariz, G.-M., Lindberg, M., Hariz, M.I. and Bergenheim, T.A. (2003) Gender Differences in Disability and HealthRelated Quality of Life in Patients with Parkinson's Disease Treated with Stereotactic Surgery. Acta Neurologica Scandinavica, 108, 28-37. http://dx.doi.org/10.1034/j.1600-0404.2003.00092.x

[15] Perez-Lloret, S., Nègre-Pagès, L., Ojero-Senard, A., Damier, P., Destée, A., Tison, F., et al. (2012) Oro-Buccal Symptoms (Dysphagia, Dysarthria, and Sialorrhea) in Patients with Parkinson's Disease: Preliminary Analysis from the French COPARK Cohort. European Journal of Neurology, 19, 28-37. http://dx.doi.org/10.1111/j.1468-1331.2011.03402.x

[16] Riedel, O., Klotsche, J., Spottke, A., Deuschl, G., Förstl, H., Henn, F., et al. (2008) Cognitive Impairment in 873 Patients with Idiopathic Parkinson's Disease. Results from the German Study on Epidemiology of Parkinson's Disease with Dementia (GEPAD). Journal of Neurology, 255, 255-264. http://dx.doi.org/10.1007/s00415-008-0720-2

[17] Solimeo, S. (2008) Sex and Gender in Older Adults' Experience of Parkinson's Disease. The Journals of Gerontology Series B: Psychological Sciences and Social Sciences, 63, S42-S48. http://dx.doi.org/10.1093/geronb/63.1.s42

[18] Hoehn, M. and Yahr, M.D. (1967) Parkinsonism: Onset, Progression and Mortality. Neurology, 17, 427-442. http://dx.doi.org/10.1212/WNL.17.5.427

[19] Armstrong, M.J., Gruber-Baldini, A.L., Reich, S.G., Fishman, P.S., Lachner, C., Shulman, L.M. (2014) Which Features of PD Predict Earlier Exit from the Workforce? Parkinsonism and Related Disorders, 20, 1257-1259. http://dx.doi.org/10.1016/j.parkreldis.2014.08.005

[20] Hughes, T.A., Ross, H.F., Musa, S., Bhattacherjee, S., Nathan, R.N., Mindham, R.H.S. and Spokes, E.G.S. (2000) A 10-Year Study of the Incidence of and Factors Predicting Dementia in Parkinson's Disease. Neurology, 54, 1596-1603. http://dx.doi.org/10.1212/WNL.54.8.1596

[21] Breteler, M., de Groot, R., Romunde, L. and Hofman, A. (1995) Risk of Dementia in Patients with Parkinson's Disease, Epilepsy, and Severe Head Trauma: A Register-Based Follow-Up Study. American Journal of Epidemiology, 142, 1300-1305.

[22] Aarsland, D., Andersen, K., Larsen, J.P., Lolk, A., Nielsen, H. and Kragh-Sørensen, P. (2001) Risk of Dementia in Parkinson's Disease. Neurology, 56, 730-736. http://dx.doi.org/10.1212/WNL.56.6.730

[23] Mayeux, R., Denaro, J., Hemenegildo, N., Marder, K., Tang, M.-X., Cote, L.J. and Stern, Y. (1992) A PopulationBased Investigation of Parkinson's Disease with and without Dementia. Archives of Neurology, 49, 492-497. http://dx.doi.org/10.1001/archneur.1992.00530290076015 